\title{
Pilot experimentation of the accuracy of accelerometer activity count-derived breaks in sedentary time
}

\author{
Paul D. Loprinzi*, Meghan K Edwards
}

Department of Health, Exercise Science and Recreation Management, School of Applied Sciences, The University of Mississippi, 229 Turner Center, University, MS 38677, USA

\begin{abstract}
Introduction: The purpose of this study was to conduct a pilot experimentation of the accuracy of the ActiGraph GT9X model in detecting breaks from sedentary behavior.

Methods: Participants completed six transitional-based sedentary break activities while wearing an ActiGraph GT9X accelerometer. Data were processed using both the default filter and low-frequency extension filter. Direct observation was used as the criterion to observe a break in sedentary behavior. Trial 1 included the transition from sitting to standing; trial 2, sitting to walking for $30 \mathrm{~s}$; trial 3, sitting to walking for 1 min; trial 4, standing to walking for 30 s; trial 5, standing to walking for 1 min; and trial 6, standing to sitting.
\end{abstract}

Results: For both the default and low-frequency filter, the accelerometer only detected a break $10 \%$ of the time for trial 1. Accuracy was also low (<40\%) for trial 6 . However, accuracy was perfect $(100 \%)$ for trials $2-5$.

Conclusion: The ActiGraph GT9X accelerometer was inaccurate in detecting a sedentary break when transitioning from a sitting to standing position but was accurate for other transitional shifts.

Key words: Accelerometry; break; exercise; physical activity

\section{INTRODUCTION}

Sedentary behavior has previously been defined as energy expenditure between 1 and 1.5 metabolic

\footnotetext{
*Corresponding author: Paul D. Loprinzi, Physical Activity Epidemiology Laboratory, Exercise Psychology Laboratory, Department of Health, Exercise Science and Recreation Management, School of Applied Sciences, The University of Mississippi, 229 Turner Center, University, MS 38677, USA. E-mail: pdloprin@olemiss.edu
}

Submitted: 19 March 2018/Accepted: 19 April 2018

DOI: https://doi.org/10.17532/jhsci.2017.514 equivalent units while sitting or lying (1), and is associated with unfavorable health outcomes (e.g., reduced aerobic capacity, muscle strength/mass, and metabolic function) (2), independent of participation in physical activity (3). For example, markers of sedentary behavior (e.g., television viewing or sitting time) have been shown to associate positively with many metabolic risk factors (e.g., elevated insulin levels), cardiovascular disease (CVD) risk and CVDrelated events, and all-cause mortality (4-8).

Mechanistically, physical inactivity stimuli may induce a short-lived inhibitor protein involved in the 
regulation of lipoprotein lipase (LPL) (9), an essential enzyme for the breakdown of triglycerides in circulating lipoproteins (10). In addition, sedentary behavior may result in the suppression of capillary LPL in muscle tissue $(11,12)$. Low LPL levels have been associated with reduced plasma high-density lipoprotein and lowered plasma triglyceride uptake $(13,14)$, both of which are risk factors for CVD $(15,16)$. Evidence of a positive association between physical activity and telomere length (17-19) makes it plausible that sedentary behavior may contribute to telomere shortening.

Corroborating the purposed associations of sedentary behavior with deleterious health outcomes, research has shown that sedentary behavior breaks (i.e., interruptions in prolonged bouts of sedentary behavior) are favorably associated with health benefits including a decreased metabolic risk (e.g., lowest waist circumference among those with the highest amount of sedentary behavior breaks) $(20,21)$. Notably, repeated exposures to bouts of prolonged sitting are thought to promote inauspicious metabolic adaptations (21). Presenting further reason to advocate against prolonged sitting, breaking up sedentary behavior more frequently may lead to higher energy expenditure (21). The aforementioned is plausible, given that the act of standing more throughout the day (as opposed to sitting) may result in significantly higher energy expenditure (22). Taken together, the previous evidence underscores the importance of minimizing sedentary behavior.

Along with working to better understand the mechanisms and consequences of sedentary behavior is the apparent need for an accurate measurement of sedentary behavior (besides direct observation). The majority of sedentary behavior studies have used self-reported assessments to examine the associations between sedentary behavior and health outcomes $(23,24)$. Notably, self-report assessments of physical activity are accompanied by numerous limitations (25) (e.g., social desirability bias and inaccurate recall) (26). In addition, the validation studies for self-reported sedentary behavior lack consistency with regard to the utilized criterion measurement, modes of administration, and target populations. Further highlighting the need for the use of more valid measurements of sedentary behavior, a review on the validity and reliability of measures of TV viewing time and non-occupational sedentary behavior (24) found that only three, of 60 identified studies, examined the validity of the employed questionnaire.

Given the limitations mentioned above of self-reported activity questionnaires, several objective measurement tools (e.g., ActiGraph activity monitor and activPAL) have been employed in efforts to provide more accurate and reliable assessments of sedentary behavior. In 2011, the hip-mounted, uniaxial ActiGraph accelerometer (model 7164) was reported (27) to be the most widely used accelerometer for free-living physical activity and sedentary behavior research in population-based studies, most commonly employing a 1-min epoch length (28). The ActiGraph activity monitor has previously been validated against both IDEEA monitor and the activPAL as criterion measures, demonstrating moderate correlations $(r=0.59$ (29) and $r=0.76$, respectively) (27), suggesting that the ActiGraph activity monitor may provide useful and accurate measures of sedentary time.

A break in sedentary behavior, as determined through accelerometry, is commonly defined as a timestamped transition from a relative lack of movement $(<100$ counts $/ \mathrm{min})$ to relatively more movement ( $\geq 100$ counts/min). This break in sedentary behavior is often considered as a postural change from sitting to standing $(21,30)$. Notably, the existing validation research comparing objective devices for monitoring such breaks is unsubstantial. Here, we highlight two studies that conducted free-living assessments of sedentary behavior breaks. In 2012, Lyden et al. (31) demonstrated that the ActiGraph GT3X, when compared to the criterion of direct observation, was not accurate in estimating sedentary behavior break rate (the number of breaks taken per sedentary hour) or an absolute number of breaks. Specifically, the ActiGraph monitor was found to significantly overestimate breaks sedentary behavior break rate and total breaks in sedentary behavior when using both a low-frequency extension filter and the normal filter, as well when employing a $100 \mathrm{cpm}$ and $150 \mathrm{cpm}$ cut point (32) for sedentary time. Similarly, Barreira et al. (30) demonstrated that the ActiGraph GT3X and the activPAL activity monitors did not correspond with regard to the number of estimated breaks in sedentary 
behavior (measured in a 7-day free-living condition). Notably, the activPAL was designed with the intent of measuring free-living activity and has the ability to differentiate among various postures and distinguish sitting, standing, and stepping activities $(33,34)$, and has previously been suggested to be a more valid tool for assessing sedentary behavior (as opposed to the ActiGraph GT3X, when using direct observation as the criterion measurement) (32). In the ActiGraph GT3X versus activPAL comparison study by Barreira et al. (30), the ActiGraph GT3X detected an average of 74 breaks/day while the activPAL detected an average of 39 breaks/day, with the ActiGraph accelerometer only detecting $67 \%$ of the breaks recognized by the activPAL, and $65 \%$ of the ActiGraph breaks not corresponding with activPAL breaks. Notably, most (52\%) of the non-corresponding ActiGraph-detected breaks occurred when participants were sitting or standing (42\%).

The present study is an extension of free-living accelerometer-based sedentary behavior measurement studies and employed a controlled experimental design to examine the accuracy of the most recent ActiGraph accelerometer model, the GT9X, in detecting breaks from sedentary behavior. Specifically, we were interested in exploring the accuracy of the ActiGraph GT9X in detecting transitions between sitting/standing, sitting/walking, and standing/walking. We recognize that standing is not typically considered a sedentary behavior, but given the difficulty for accelerometers to distinguish between sitting and standing (35), we felt this would also be a worthwhile "sedentary break" to investigate. Further, we employ the ActiGraph accelerometer for this study given that, at the time of this writing, it is the most widely used accelerometer in sedentary behavior and physical activity research.

\section{METHODS}

\section{Participants and measurement of breaks}

Ten university students were asked to participate in this pilot investigation. Participant consent was obtained before data collection and the author's institutional review board approved all study procedures. During all conditions described below, participants wore an ActiGraph GT9X accelerometer on their right hip at the midaxillary line, with direct observation serving as the criterion measure. Raw ActiGraph GT9X data were collected at $30 \mathrm{~Hz}$, integrated using a 60-s epoch (to increase the generalizability of real-world free-living studies), and filtered using both the low-frequency extension filter and the default filter. The ActiLife software was used to evaluate the detection of the accelerometer-assessed sedentary break. Within this software, the Sedentary Analysis Options Editor macro was modified by applying the sedentary minimum length to be $1 \mathrm{~min}$ and the count level/min minimum and maximum, respectively, to be 0 and 99 .

\section{Experimental conditions}

1. Participants sat for $3 \mathrm{~min}$ at a computer, then immediately stood for 3 min while working on their computer.

2. Participants sat for $3 \mathrm{~min}$ at their computer, then immediately walked at a low intensity (in a hallway) for $30 \mathrm{~s}$.

3. Participants sat for $3 \mathrm{~min}$ at their computer, then immediately walked at a low intensity (in a hallway) for $1 \mathrm{~min}$.

4. Participants stood for $3 \mathrm{~min}$ at their computer, then immediately walked at a low intensity (in a hallway) for $30 \mathrm{~s}$.

5. Participants stood for $3 \mathrm{~min}$ at their computer, then immediately walked at a low intensity (in a hallway) for $1 \mathrm{~min}$.

6. Participants stood for $3 \mathrm{~min}$ at their computer, then immediately sat for $3 \mathrm{~min}$.

\section{Analysis}

The percentage of ActiGraph-detected sedentary breaks from the above six conditions was compared to the criterion of direct observation. Estimates were computed for both the low-frequency extension filter and the default filter.

\section{RESULTS}

Table 1 reports the demographic characteristics of the analyzed sample. Participants, on average, were 27 years, with $60 \%$ being male. Table 2 displays the proportion of sedentary breaks detected by accelerometry during the transitional trials. Except for trial 6 , results were similar when applying the default 
filter versus low-frequency filter. The accelerometer detected the transitional break $100 \%$ of the time for trials 2-5. However, a low percentage of sedentary break detection was observed for trials 1 and 6. For both the default and low-frequency filter, the accelerometer only detected a break $10 \%$ of the time for trial 1 , which was when the participant transitioned from a sitting to standing position. Similarly, 20\% (default)-40\% (low-frequency filter) of the time, the accelerometer correctly detected a break for trial 6 when participants transitioned from standing to sitting.

\section{DISCUSSION}

The purpose of this pilot study was to evaluate the ability of the ActiGraph GT9X to accurately detect a break in sedentary behavior during various transitional-based sedentary break activities. The motivation for this investigation resulted from the emerging body of work demonstrating a favorable association between sedentary break frequency and various cardiometabolic-related parameters $(20,21)$. The main finding of this study was that

TABLE 1. Demographic characteristics of the analyzed sample $(n=10)$

\begin{tabular}{lcc}
\hline Variable & Point estimate & $95 \% \mathrm{Cl}$ \\
\hline Age, mean years & 27.2 & $25.0-29.3$ \\
Gender, \% male & 60 & \\
Race-ethnicity, \% & & \\
$\quad$ non-Hispanic white & 80 & \\
non-Hispanic black & 10 & \\
$\quad$ Others & 10 & \\
Body mass index, mean kg/m ${ }^{2}$ & 25.1 & $23.4-26.7$ \\
Waist circumference, mean cm & 83.3 & $76.8-89.6$ \\
\hline
\end{tabular}

$\mathrm{Cl}$ : Confidence interval the ActiGraph GT9X accelerometer was a $100 \%$ accurate detection of "sedentary breaks" for all of the trials that resulted in walking, including two sitting-to-walking trials and two standing-to-walking trials. However, the monitor infrequently detected a transition when going from sitting to standing ( $10 \%$ of the time) or standing to sitting (20-40\% of the time).

Few studies have evaluated the sensitivity of accelerometry to detect sedentary breaks $(30,31)$. Lyden et al. (31) evaluated 13 participants during a free-living setting. Compared to direct observation, they concluded that the ActiGraph GT3X accelerometer was not accurate in estimating break rate or the absolute number of breaks. Similarly, during a free-living assessment, Barreira et al. (30) demonstrated that the ActiGraph GT3X and the activPAL accelerometers did not correspond with regard to the number of estimated breaks in sedentary behavior. On average, the ActiGraph GT3X detected 67\% of the activPAL breaks. Of the non-corresponding ActiGraph breaks, 52\% occurred when participants were sitting, $42 \%$ when standing, and $6 \%$ when transitioning from standing to sitting. In partial agreement with these studies, the present study demonstrated very poor detection of a sedentary break during the transitions from sitting to standing as well as a poor detection of a sedentary break from standing to sitting. However, in the present study, when the participant walked for at least $30 \mathrm{~s}$ after transitioning from a sedentary behavior, there was perfect accuracy in detecting the break. Of course, no study is without limitations. The present study was limited by a relatively small sample size $(n=10)$; however, similar sample sizes $(n=13-15)(30,31)$ have been utilized in previous sedentary behavior break research and are common in accelerometer

TABLE 2. The proportion of sedentary breaks detected by accelerometry during the transitional trials

\begin{tabular}{|c|c|c|c|}
\hline \multirow[t]{2}{*}{ Trial } & \multirow[t]{2}{*}{ Description } & \multicolumn{2}{|c|}{$\begin{array}{c}\% \text { sedentary breaks detected by } \\
\text { accelerometry }\end{array}$} \\
\hline & & Default filter & Low-frequency filter \\
\hline 1 & Sat for 3 min at a computer, then immediately stood for 3 min while working on their computer & 10 & 10 \\
\hline 2 & Sat for $3 \mathrm{~min}$ at their computer, then immediately walked at a low intensity for $30 \mathrm{~s}$ & 100 & 100 \\
\hline 3 & Sat for $3 \mathrm{~min}$ at their computer, then immediately walked at a low intensity for 1 min & 100 & 100 \\
\hline 4 & Stood for $3 \mathrm{~min}$ at their computer, then immediately walked at a low intensity for $30 \mathrm{~s}$ & 100 & 100 \\
\hline 5 & Stood for $3 \mathrm{~min}$ at their computer, then immediately walked at a low intensity for 1 min & 100 & 100 \\
\hline 6 & Stood for $3 \mathrm{~min}$ at their computer, then immediately sat for $3 \mathrm{~min}$ & 20 & 40 \\
\hline
\end{tabular}


studies $(26,35)$. This study is strengthened by its novelty, the use of the latest version of the ActiGraph accelerometer, and the inclusion of multiple types of sedentary breaks.

The ActiGraph GT9X's low accuracy in detecting sedentary behavior breaks when transitioning between sitting and standing positions may have important implications, given the widespread use of ActiGraph accelerometers for population-based studies (28) as well as the emergence of work evaluating the efficacy of sit-stand workstations (36). If a significant proportion of an individual's day includes transitioning between sitting and standing, it is possible that the findings of previous work evaluating the associations between sedentary breaks and health-enhancing cardiometabolic parameters have been overestimated. That is, free-living assessments of sedentary break frequency, according to our results, may have grossly underestimated the number of breaks from sitting to standing, suggesting that more breaks actually took place to elicit the observed health outcomes (i.e., a weaker per break "effect" on health outcomes). Given the results of the current study, it is recommended that future intervention-based studies evaluating the health effects of sedentary behavior breaks should include some brief ambulation with each break to elicit the most accurate detection of the breaks, especially if administered in the free-living setting. For example, if participants wearing accelerometers in a free-living setting are given instructions to break up their sitting every hour, they should be instructed to not merely stand up, but to stand and walk for at least $30 \mathrm{~s}$. Further, the ActiGraph accelerometer, in particular, could be refined to be more sensitive in detecting sedentary breaks that result in a transition from sitting to standing. One possible way, this could be done, is to integrate counts per minute along with inclinometer data. Ultimately, numerous additional randomized controlled trials are needed to determine (with more certainty) the accuracy of commonly employed measurement tools for detecting breaks in sedentary behaviors before we can make confident conclusions regarding the extent of the health benefits that may emerge due to breaking up prolonged sitting.

\section{REFERENCES}

1. Pate RR, O'Neill JR, Lobelo F. The evolving definition of "sedentary". Exerc Sport Sci Rev 2008;36:173-8.

https://doi.org/10.1097/JES.0b013e3181877d1a.

2. Thyfault JP, Du M, Kraus WE, Levine JA, Booth FW. Physiology of sedentary behavior and its relationship to health outcomes. Med Sci Sports Exerc 2015;47:1301-5.

https://doi.org/10.1249/MSS.0000000000000518.

3. Owen N, Sparling PB, Healy GN, Dunstan DW, Matthews CE. Sedentary behavior: Emerging evidence for a new health risk. Mayo Clin Proc 2010;85:1138-41

https://doi.org/10.4065/mcp.2010.0444.

4. Healy GN, Wijndaele K, Dunstan DW, Shaw JE, Salmon J, Zimmet PZ, et al. Objectively measured sedentary time, physical activity, and metabolic risk: The Australian diabetes, obesity and lifestyle study (AusDiab). Diabetes Care 2008:31:369-71.

https://doi.org/10.2337/dc07-1795.

5. Dunstan DW, Barr EL, Healy GN, Salmon J, Shaw JE, Balkau B, et al. Television viewing time and mortality: The Australian diabetes, obesity and lifestyle study (AusDiab). Circulation 2010;121:384-91.

https://doi.org/10.1161/CIRCULATIONAHA.109.894824.

6. Stamatakis E, Hamer M, Dunstan DW. Screen-based entertainment time, allcause mortality, and cardiovascular events: Population-based study with ongoing mortality and hospital events follow-up. J Am Coll Cardiol 2011;57:292-9.

https://doi.org/10.1016/j.jacc.2010.05.065.

7. Weller I, Corey P. The impact of excluding non-leisure energy expenditure on the relation between physical activity and mortality in women. Epidemiology 1998:9:632-5.

https://doi.org/10.1097/00001648-199811000-00012.

8. Manson JE, Greenland $\mathrm{P}$, LaCroix $\mathrm{AZ}$, Stefanick ML, Mouton $\mathrm{CP}$, Oberman $A$, et al. Walking compared with vigorous exercise for the prevention of cardiovascular events in women. N Engl J Med 2002;347:716-25.

https://doi.org/10.1056/NEJMoa021067.

9. Hamilton MT, Hamilton DG, Zderic TW. Exercise physiology versus inactivity physiology: An essential concept for understanding lipoprotein lipase regulation. Exerc Sport Sci Rev 2004;32:161-6.

https://doi.org/10.1097/00003677-200410000-00007.

10. Wittrup HH, Tybjaerg-Hansen A, Nordestgaard BG. Lipoprotein lipase mutations, plasma lipids and lipoproteins, and risk of ischemic heart disease. A meta-analysis. Circulation 1999;99:2901-7.

https://doi.org/10.1161/01.CIR.99.22.2901.

11. Bey L, Hamilton MT. Suppression of skeletal muscle lipoprotein lipase activity during physical inactivity: A molecular reason to maintain daily low-intensity activity. J Physiol 2003;551:673-82.

https://doi.org/10.1113/jphysiol.2003.045591.

12. Hamilton MT, Etienne J, McClure WC, Pavey BS, Holloway AK. Role of local contractile activity and muscle fiber type on LPL regulation during exercise. Am J Physiol 1998;275:E1016-22.

13. Herd SL, Kiens B, Boobis LH, Hardman AE. Moderate exercise, postprandial lipemia, and skeletal muscle lipoprotein lipase activity. Metabolism 2001;50:756-62.

https://doi.org/10.1053/meta.2001.24199.

14. Goldberg IJ, Le NA, Ginsberg HN, Krauss RM, Lindgren FT. Lipoprotein metabolism during acute inhibition of lipoprotein lipase in the cynomolgus monkey. J Clin Invest 1988;81:561-8.

https://doi.org/10.1172/JCl113354.

15. Toth PP. High-density lipoprotein and cardiovascular risk. Circulation 2004;109:1809-12. 
https://doi.org/10.1161/01.CIR.0000126889.97626.B8.

16. Miller M, Stone NJ, Ballantyne C, Bittner V, Criqui MH, Ginsberg HN, et al. Triglycerides and cardiovascular disease: A scientific statement from the american heart association. Circulation 2011;123:2292-333.

https://doi.org/10.1161/CIR.0b013e3182160726.

17. Loprinzi PD, Sng E. Mode-specific physical activity and leukocyte telomere length among U.S. Adults: Implications of running on cellular aging. Prev Med 2016;85:17-9.

https://doi.org/10.1016/j.ypmed.2016.01.002.

18. Loprinzi PD, Loenneke JP, Blackburn EH. Movement-based behaviors and leukocyte telomere length among US adults. Med Sci Sports Exerc 2015;47:2347-52.

\section{https://doi.org/10.1249/MSS.0000000000000695.}

19. Du M, Prescott J, Kraft P, Han J, Giovannucci E, Hankinson SE, et al. Physical activity, sedentary behavior, and leukocyte telomere length in women. Am J Epidemiol 2012;175:414-22.

https://doi.org/10.1093/aje/kwr330.

20. Saunders TJ, Tremblay MS, Mathieu MĖ, Henderson M, O'Loughlin J, Tremblay A, et al. Associations of sedentary behavior, sedentary bouts and breaks in sedentary time with cardiometabolic risk in children with a family history of obesity. PLoS One 2013;8:e79143.

https://doi.org/10.1371/journal.pone.0079143.

21. Healy GN, Dunstan DW, Salmon J, Cerin E, Shaw JE, Zimmet PZ, et al. Breaks in sedentary time: Beneficial associations with metabolic risk. Diabetes Care 2008;31:661-6.

https://doi.org/10.2337/dc07-2046.

22. Levine JA. Nonexercise activity thermogenesis (NEAT): Environment and biology. Am J Physiol Endocrinol Metab 2004;286:E675-85.

https://doi.org/10.1152/ajpendo.00562.2003.

23. Atkin AJ, Gorely T, Clemes SA, Yates T, Edwardson C, Brage S, et al. Methods of measurement in epidemiology: Sedentary behaviour. Int $\mathrm{J}$ Epidemiol 2012;41:1460-71.

https://doi.org/10.1093/ije/dys118.

24. Clark BK, Sugiyama T, Healy GN, Salmon J, Dunstan DW, Owen N, et al. Validity and reliability of measures of television viewing time and other non-occupational sedentary behaviour of adults: A review. Obes Rev 2009;10:7-16.

https://doi.org/10.1111/j.1467-789X.2008.00508.x.

25. Sallis JF, Saelens BE. Assessment of physical activity by self-report: Status, limitations, and future directions. Res Q Exerc Sport 2000;71:S1-14. https://doi.org/10.1080/02701367.2000.11082780.

26. Prince SA, Adamo KB, Hamel ME, Hardt J, Connor Gorber S, Tremblay M, et al. A comparison of direct versus self-report measures for assessing physical activity in adults: A systematic review. Int J Behav Nutr Phys Act 2008:5:56.

https://doi.org/10.1186/1479-5868-5-56.

27. Healy GN, Clark BK, Winkler EA, Gardiner PA, Brown WJ, Matthews CE, et al. Measurement of adults' sedentary time in population-based studies. Am J Prev Med 2011;41:216-27.

https://doi.org/10.1016/j.amepre.2011.05.005.

28. Hagströmer M, Oja P, Sjöström M. Physical activity and inactivity in an adult population assessed by accelerometry. Med Sci Sports Exerc 2007;39:1502-8.

https://doi.org/10.1249/mss.0b013e3180a76de5.

29. Matthews CE, Chen KY, Freedson PS, Buchowski MS, Beech BM, Pate RR, et al. Amount of time spent in sedentary behaviors in the United States, 2003-2004. Am J Epidemiol 2008;167:875-81.

https://doi.org/10.1093/aje/kwm390.

30. Barreira TV, Zderic TW, Schuna JM Jr., Hamilton MT, Tudor-Locke C. Freeliving activity counts-derived breaks in sedentary time: Are they real transitions from sitting to standing? Gait Posture 2015;42:70-2.

https://doi.org/10.1016/j.gaitpost.2015.04.008.

31. Lyden K, Kozey Keadle SL, Staudenmayer JW, Freedson PS. Validity of two wearable monitors to estimate breaks from sedentary time. Med Sci Sports Exerc 2012;44:2243-52.

https://doi.org/10.1249/MSS.0b013e318260c477.

32. Kozey-Keadle S, Libertine A, Lyden K, Staudenmayer J, Freedson PS. Validation of wearable monitors for assessing sedentary behavior. Med Sci Sports Exerc 2011;43:1561-7.

https://doi.org/10.1249/MSS.0b013e31820ce174.

33. Grant PM, Ryan CG, Tigbe WW, Granat MH. The validation of a novel activity monitor in the measurement of posture and motion during everyday activities. Br J Sports Med 2006;40:992-7.

https://doi.org/10.1136/bjsm.2006.030262.

34. Ryan CG, Grant PM, Tigbe WW, Granat MH. The validity and reliability of a novel activity monitor as a measure of walking. $\mathrm{Br} \mathrm{J}$ Sports Med 2006; $40: 779-84$.

https://doi.org/10.1136/bjsm.2006.027276.

35. Lugade V, Fortune E, Morrow M, Kaufman K. Validity of using tri-axial accelerometers to measure human movement - Part I: Posture and movement detection. Med Eng Phys 2014;36:169-76.

https://doi.org/10.1016/j.medengphy.2013.06.005.

36. Shrestha N, Kukkonen-Harjula KT, Verbeek JH, ljaz S, Hermans V, Pedisic Z, et al. Workplace interventions for reducing sitting at work. Cochrane Database Syst Rev 2018;6:CD010912.

https://doi.org/10.1002/14651858.CD010912.pub4. 